\title{
Les déterminants de l'innovation dans une économie africaine à revenu intermédiaire, une réévaluation des modèles d'innovation ${ }^{1}$
}

\section{The drivers of innovation in an African middle-income economy, a reassessment of innovation models}

\author{
Christian Le Bas ${ }^{1}$, Laurence Nkakene Molou² \\ ${ }^{1}$ Esdes, UCLy, France, clebas@univ-catholyon.fr \\ ${ }^{2}$ Centre d'Etudes et de Recherche en Economie et Gestion (CEREG), Université de Yaoundé II, Cameroun, \\ molou_l@yahoo.fr
}

RÉSUMÉ. Les recherches sur l'innovation dans les économies africaines ne sont pas nombreuses. Nous contribuons à la littérature sur les déterminants (drivers) de l'innovation de plusieurs façons. En premier lieu, nous partons de l'hypothèse que les économies africaines sont très diverses et qu'en conséquence le jeu complexe des drivers sur le comportement d'innovation doit être apprécié selon le type d'économie. Nous distinguons plusieurs catégories d'économies africaines et nous focalisons nos recherches sur les économies africaines à revenu intermédiaire, notamment le Cameroun. En second, lieu nous prenons le comportement d'innovation dans sa diversité (produit/procédé ; simple/complexe). Les estimations économétriques, à partir données de la banque Mondiale pour l'année 2016, tendent à montrer que les dépenses de R-D n'expliquent pas la décision d'innover. Ce résultat très peu néo-schumpetérien pourrait s'expliquer par trois ensembles de facteurs: l'existence d'un modèle d'innovateur atypique de type market pull ; la présence d'innovation dite below the radar, l'importance d'innovation frugale.

ABSTRACT. There are few studies dealing with the drivers of innovation in African economies. We contribute to the literature in many ways. First of all, due to the diversity of African economies, we argue it is necessary to target the type of economy we observe. As a consequence, we focus our research on the African economies with medium level revenue, namely Cameroon. Secondly, we analyze the innovation behavior by distinguishing product versus process innovator and single versus complex innovator. Thirdly we use the data set released by the World Bank for 2016. Our estimations show the R\&D expenditures do not explain the innovation decision. This result that contrasts with the Schumpeterian (evolutionary) tradition could be accounted for by three factors: the existence of a market pull strategy, the presence of innovations "below the radar", the importance of frugal and grassroots innovations.

MOTS CLÉS. économies africaines, déterminants de l'innovation, dépenses de R-D, innovation frugale.

KEY WORDS. African economies, innovation drivers, R\&D expenditures, frugal and grassroots innovation.

\section{Introduction}

Les recherches sur l'innovation dans les économies africaines ne sont pas nombreuses. Peu se concentrent sur les facteurs qui déterminent les entreprises à construire un comportement d'innovation technologique (Adeyeye et al., 2015 ; Casadella et Temple, 2016 ; Egbetokun et al., 2009). Nous contribuons à la littérature sur les déterminants (drivers) de l'innovation en économies africaines de plusieurs façons. En premier lieu, nous partons de l'hypothèse que les économies africaines sont très diverses et qu'en conséquence le jeu complexe des drivers sur le comportement d'innovation doit être apprécié selon le type d'économie. Nous distinguons plusieurs catégories d'économies africaines et nous focalisons nos recherches sur les économies africaines à revenu intermédiaire. L'économie choisie est celle du Cameroun. En second lieu nous prenons le comportement d'innovation dans sa diversité.

\footnotetext{
${ }^{1}$ Nous remercions N. Poussing du Liser (Luxembourg) pour son aide.
} 
Traditionnellement, on retient le comportement d'innovation de produit opposé à l'innovation de procédé (Fagerberg et al., 2004; Swann, 2009). Cette distinction est importante mais nous pousserons plus l'analyse en investiguant la dualité innovateur simple/innovateur complexe qui s'est imposé récemment (voir notamment Le Bas et Poussing, 2014; Tavassoli et Karlsson, 2015). Les inventeurs simples investissent dans une seule activité d'innovation (produit ou process) au cours de la même période de temps. Les deux types d'innovation renvoient à des types de concurrence et à des types de stratégies différentes.

Les stratégies d'innovation de process sont associées à un régime de concurrence par les prix (Pianta, 2005). En revanche, le comportement d'innovation de produit, qui renvoie à plus de densité, est lié à la compétition technologique entre firmes à la recherche du leadership technologique. L'innovation de produit doit être considérée comme importante dans le contexte des économies africaines. En effet, elle peut déboucher sur ce que Chataway et ses collègues appellent une propoor innovation (Chataway et al., 2014), c'est-à-dire une innovation faite pour inclure les catégories les plus pauvres dans le processus d'achat et de consommation de biens et services (l'innovation inclusive $)^{2}$.

Les innovateurs complexes mènent les deux activités de front dans la même période. La complexité vient du fait qu'il y a des interactions de connaissances entre les deux types d'innovation (spillovers de connaissance qui vont dans les deux sens). Il reste vrai que la taille des firmes ici joue un rôle : une grande firme possède assez de ressources pour mener les deux activités dans le même temps. Il y a complexité car il existe des relations interactives entre les technologies de produits et celles relatives aux processus. Les avantages des innovateurs complexes se déploient donc dans les deux dimensions. Leurs nouveaux produits (ou améliorés) leurs ouvrent de nouveaux marchés (avantage crucial). Ainsi, les innovations de réduction de coûts permettent un détournement de la demande à leur profit. Les deux types d'innovation produisent in fine une meilleure rentabilité. En dernier lieu, nous explorons les (plutôt récentes) analyses non schumpetériennes de l'innovation en rapport avec les contextes des économies africaines.

Le papier se lit comme suit. Le contexte économique des économies africaines à revenu intermédiaire et leur capacité d'innovation sont d'abord décrits. Ce que nous dit la littérature sur les déterminants de l'innovation est passé ensuite en revue. On mettra également l'accent sur ce que l'on doit retenir pour l'analyse du cas africain. On s'intéresse ensuite aux données de notre analyse empirique, à la description de l'échantillon et à la définition des variables. Les modèles estimés et la mise en évidence des résultats que nous retiendrons. On se propose ensuite d'interpréter les résultats en présentant des schémas adaptés au cas africain du processus d'innovation sans recherche et développement (R-D). En conclusion, on tire quelques enseignements pour le développement économique.

\section{Le contexte économique des économies africaines à revenu intermédiaire et leur capacité d'innovation}

Notre hypothèse fondatrice est que les performances à l'innovation des firmes et leur caractère plus ou moins soutenable dépendent des caractéristiques structurelles des économies africaines. Celles-ci diffèrent grandement d'une économie à l'autre ${ }^{3}$. Cela rend nécessaire de fournir une typologie des économies du continent africain. On s'appuie ici sur le travail de Jacquemot (2013) distinguant 4 types d'économies africaines : les économies à faible revenu ; les économies à revenu

\footnotetext{
${ }^{2}$ Ce point sera repris ultérieurement quand on discutera des résultats (section 6).

${ }^{3}$ Cela sera explicité plus longuement à la page 11 et les pages suivantes. 
intermédiaire ; les économies diversifiés et les économies rentières. Il est important de bien spécifier que dans chacune des économies le moteur de la croissance est différent ${ }^{4}$.

Les économies rentières tirent un important surplus de la vente sur les marchés mondiaux de ressources de leur sous-sol. Ce surplus aux mains de la puissance publique peut constituer un levier de développement via des politiques économiques et industrielles adaptées. Les économies à revenu intermédiaire ne disposent pas de ce surplus. Leur dynamique de croissance est de type agro exportateur (Jacquemot, 2013). Le Cameroun appartient à cette seconde catégorie avec un revenu par tête de 800 à 1200 dollars en 2012. Dans le cas du Cameroun, l'exportation du bois constitue une activité essentielle. L'huile de palme constitue l'autre grand produit d'exportation. Au cours des années 1970-80, les économies à revenu intermédiaire ont vu un début d'import-substitution. Autrement dit, des industries locales ont substitué leur fabrication à des importations. Certaines économies africaines de ce type ont fait l'expérience d'une croissance rapide ces 15 dernières années comme le Botswana ou le Ghana (sur ce point voir Diao et al., 2017). Aussi serait-il fallacieux de toujours considérer ces économies comme inaptes à la croissance économique.

Toutefois, d'autres facteurs macro-économiques rendent difficiles les retombées économiques de l'innovation dans les pays africains (voir notamment Christensen et al., 2017) : une corruption omniprésente, des infrastructures insuffisamment fiables, une classe moyenne émergente qui ne peut être le levier de l'expansion des marchés de biens de consommation. Bien que ces caractéristiques s'appliquent à beaucoup d'économies africaines, elles semblent jouer un rôle plus important dans les économies à revenu intermédiaire et s'ajoutent au manque de compétences.

La question de la croissance et de la durabilité de l'entreprise formelle, celle qui nous intéresse ici, prend un sens particulier dans ce type d'économie africaine. Le projet d'entreprise ouvre un chemin couvert d'embuches (Jacquemot, 2013 : 313). Les facteurs de succès et d'échecs sont autant économiques que culturels. Par ailleurs, les firmes multinationales ont connu beaucoup de déboires et ont globalement renoncé à investir dans les marchés de consommation de beaucoup de pays africains compte tenu de la corruption. En butte à la corruption, les entreprises locales ne peuvent prospérer qu'à condition de se développer sous le mode de l'intégration industrielle verticale (ce qui évite le comportement opportuniste des fournisseurs) ou au sein de structure familiale instituant des relations de confiance malgré l'émergence d'autres difficultés (Adedayo et Ojo, 2016).

Il faut néanmoins souligner que contrairement au modèle de Ranis et Fei (1961), il n'existe pas une structure économique nationale dualiste composée d'un secteur agricole à faible productivité et un secteur industriel dit moderne dont la croissance est alimentée par les gains de productivité et le changement structurel. D'une part, aujourd'hui, le secteur des services s'est densifié (les télécommunications par exemple), d'autre part, l'importance des activités «paraformelles et informelles » (Chaléard et Sanjuan, 2017) rend la thèse dualiste beaucoup moins pertinente. Enfin, le secteur moderne est sans doute beaucoup moins dynamique en termes de développement technologique par rapport aux visions fécondes mais maintenant décalées des fondateurs du dualisme. Ce point est crucial. Il s'explique, selon nous, par le fait que les déterminants de l'innovation présentent des configurations spécifiques en économies africaines.

\footnotetext{
${ }^{4}$ Les 4 types d'économies africaines sont caractérisés par des dynamiques de développement différentes. Dans les économies rentières, pétrolières et minières, les taux de croissance ont été élevés, accompagnés d'importants investissements étrangers. Le reste de l'économie est fondé sur un appareil de production précaire. Les économies à faible revenu (dont beaucoup se trouvent en zone sahélienne) sont fragiles et à prépondérance agricole. Les économies à revenu intermédiaire ont une dynamique de croissance du modèle agro-exportateur. Elles ont aussi enregistré dans le passé un début d'import-substitution. Les économies diversifiées, à revenu intermédiaire supérieur se caractérise par un poids relatif accordé au secteur manufacturier intégré à l'échange international (textiles).
} 


\section{Déterminants de l'innovation : ce que dit la littérature et ce que l'on doit retenir}

La théorie néo schumpetérienne dont le modèle focal a été construit par Nelson et Winter (1982) s'articule autour des travaux empiriques d'une approche développée par trois auteurs qu'on réunit ici sous le titre d'approche de Mansfield-Pavitt-Scherer. Elle tend à résumer près de 40 ans de recherches empiriques quant à l'impact des variables spécifiques sur le comportement d'innovation des firmes saisies dans son contexte industriel concurrentiel. Les principaux phénomènes sont l'impact de la taille des firmes et des opportunités technologiques (Mansfield, 1995), les types d'apprentissage technologique et les sources d'innovation (Pavitt, 1984), les conditions d'appropriation et les effets complexes des structures de marché (Scherer, 1991). Bien que le processus de détermination de l'innovation soit complexe et multidimensionnel le cœur du dispositif théorique est occupé par l'équation R-D/Innovation. Les dépenses de R-D sont considérées comme le déterminant fort de l'innovation en adéquation avec les schémas théoriques défendus par Nelson et Winter (1982). Le rapport aux clients et aux fournisseurs est un point central des études sur l'innovation ${ }^{5}$. Ce qui est aussi validé en contexte africain (voir le travail sur le Nigeria d'Egbetokun et al., 2009).

Dans les pays industriels avancés, un système national d'innovation dense et riche permet aux firmes de construire de solides et persistantes trajectoires d'innovation structurées par la relation dynamique et persistante R-D/Innovation. En revanche, dans les pays en développement la dynamique schumpetérienne n'existe pas (Hobday, 2005). Les entreprises opèrent dans un contexte où les infrastructures d'une économie de la connaissance (système éducatif et de ressources humaines) restent encore manquantes ou peu denses. Asongu (2014) livre même un diagnostic plus pessimiste sur les économies africaines en remarquant que de 2000 à 2009 tous les indicateurs relatifs à l'économie de la connaissance chutent. Le fait que les grandes firmes manquent de ressources technologiques internes et externes rend difficile si ce n'est impossible, l'absorption de technologies extérieures. Aussi la thèse des latecomers (voir toujours Hobday, 2005) semble s'imposer dans les pays africains à économie intermédiaire. Une conséquence importante, pour le modèle d'innovation des latecomers, est que de substantielles barrières empêchent que la R-D des firmes industrielles soit au cœur de la dynamique schumpetérienne. On doit donc s'attendre à ce que la relation $\mathrm{R}-\mathrm{D} /$ Innovation ne soit pas vérifiée dans ce type d'économies. En revanche, il y a des effets positifs des systèmes locaux de production et d'innovation, en bref des clusters (Haudeville et Le Bas, 2018). Dans beaucoup d'industries africaines de types low ou medium tech les connaissances nouvelles qui génèrent des processus innovant passent par les relations avec les acheteurs ou les fournisseurs qui constituent le canal essentiel pour l'innovation. Par exemple, dans l'industrie éthiopienne du textile, les connaissances entre acteurs sont transférées au sein des clusters informels (Gebreeyesus et Mohnen, 2013).

Ce bref survey de littérature, nous incite à retenir une hypothèse. Les activités de R-D ne sont pas toujours des déterminants de l'innovation dans les économies africaines à revenu intermédiaire. D'où :

\section{H1. Il n'y a pas de relation entre dépenses de R-D et innovation au niveau des firmes.}

Dans la tradition évolutionniste les capacités (dynamiques) d'une firme sont essentielles pour la maintenir sur un sentier soutenable de long terme (Nelson and Winter, 1982; Teece and Pisano, 1994). Dans ce contexte, le second cadre théorique qu'on peut mobiliser pour notre travail empirique est la théorie des ressources ou RBV (Wernerfelt, 1984 ; Barney, 1991) : une innovation réussie peut dépendre des compétences et des capacités spécifiques de l'entreprise. En effet, la RBV stipule qu'une entreprise acquiert un avantage concurrentiel en développant, en combinant et en

\footnotetext{
${ }^{5}$ Malheureusement, on n'aura pas les données pour valider ce point sur l'économie camerounaise.
} 
déployant efficacement ses ressources physiques, humaines et organisationnelles de manière à ajouter une valeur unique difficile à imiter pour les concurrents (Barney, 1991). Beaucoup d'arguments basés sur les ressources sont ancrés dans les ressources humaines (Wright et al., 1999 ; Wright et al., 2001). Il s'agit des compétences, des connaissances et le comportement des employés ou encore le contrôle des ressources organisationnelles notamment à travers les routines et les mécanismes d'apprentissage (Nelson et Winter, 1982).

Ainsi, les ressources qui sont rares, précieuses, inimitables et non substituables peuvent fournir des sources d'avantage concurrentiel durable. Dans cet article, l'innovation est non seulement un processus de création de connaissances mais aussi de compétences. Cependant, Wright et al. (2001) soutiennent que les ressources humaines ne constituent pas le fondement d'un avantage concurrentiel puisque toute pratique de ressource humaine individuelle peut être facilement copiée par les concurrents. Ils suggèrent que le capital humain, à travers une main d'œuvre hautement qualifiée et hautement motivée, constitue la source de l'avantage concurrentiel durable. Plus précisément, la ressource humaine doit avoir à la fois des niveaux élevés de compétence et une volonté (c'est-à-dire une motivation) d'afficher un comportement productif.

Toutefois, les entreprises qui recourent à une stratégie axée sur les ressources tentent également d'accumuler des ressources technologiques précieuses et utilisent une position de propriété intellectuelle agressive. Ceux qui réussissent sur les marchés internationaux sont ceux qui exercent une innovation de produits rapide et flexible, associée à une capacité de gestion basée sur les compétences internes et externes. À ce sujet, Teece et al. (1997) présentent cette gestion stratégique autour de l'approche des capacités dynamiques. Par conséquent, le concept de capacités dynamiques renvoie à cette capacité qui intègre, construit et reconfigure les compétences internes et externes pour aborder l'évolution rapide de l'environnement. Contrairement à la théorie des ressources qui met l'accent sur les capacités et les atouts spécifiques des entreprises.

Une seconde idée au cœur de notre dispositif est que le capital humain des entrepreneurs et des salariés constitue une ressource déterminante. Dans ce contexte le niveau de formation initiale du personnel apparaît comme une variable déterminante, décrivant la capacité de la firme à se projeter dans l'économie de la connaissance et à mener à bien des projets d'innovation. Aussi devons-nous tester l'hypothèse :

H2. La proportion de personnes diplômées joue positivement sur la capacité à mener à bien des innovations.

A côté des variables cruciales comme la R-D et le niveau de formation, beaucoup de travaux soulignent que la taille de la firme joue un rôle crucial. Une grande entreprise possède une capacité à innover plus importante. Aussi la taille représentera pour nous une variable de contrôle.

Pour Lazonick (2005), les conditions sociales de l'entreprise innovante sont importantes. Par conditions sociales, il faut entendre la définition de la stratégie, la recherche des moyens de financement et l'organisation des capacités et processus de la firme pour mener à bien les activités de production et d'innovation. Dans ce travail, la définition de la stratégie est prise en compte par les différents types d'innovation que la firme peut réaliser. L'organisation va être d'une certaine façon décrite par l'existence d'une activité de recherche et par le niveau des compétences des ressources humaines. En contrepartie, on ne traite pas dans ce travail des questions de financement de l'innovation. Le problème est, toutefois, moins sévère pour les économies africaines pour lesquelles l'innovation peut être moins coûteuse. 


\section{Les données : description de l'échantillon et définition des variables}

Pour les données de la présente recherche, nous avons eu recours à la base de données produite par la Banque Mondiale sur l'année 2016. Les données sont recueillies par les réponses à un questionnaire qui porte sur les informations générales de l'entreprise, les infrastructures et services, les ventes et approvisionnement, le niveau de concurrence, l'innovation, les capacités, les terrains et permis, la criminalité, les finances, la microfinance, les relations entre entreprises et le gouvernement, le travail, l'environnement des affaires et la performance. L'exploitation de cette base de données a permis d'avoir des informations sur le secteur d'activité des entreprises, l'âge de création des entreprises, la taille de l'entreprise, la force de travail (les travailleurs diplômés...), les dépenses de R\&D et sur l'innovation.

L'échantillon retenu pour ce travail de recherche est composé de 361 entreprises camerounaises dont 205 sont des petites entreprises (PE), 87 des moyennes (ME) et 69 des grandes (GE). Ces entreprises opèrent majoritairement dans deux secteurs d'activités, quel que soit le statut juridique : plus de 200 entreprises relèvent du commerce et de détails et les entreprises de services, 120 entreprises du secteur manufacturier. Elles sont localisées pour la plupart dans les villes. L'étude de la localisation géographique des entreprises de notre échantillon fait apparaître une forte concentration autour des pôles suivants : le Centre (41,27\% des entreprises), le Littoral $(39,61 \%)$ et l'Ouest (19,11\%). La majorité des entreprises du Cameroun sont concentrées dans 3 villes Yaoundé, Douala et Bafoussam (65\%, selon le rapport de l'Institut National de la Statistique de 2010). Notre échantillon est donc plus concentré autour de ces trois grandes villes.

\section{Variables dépendantes}

\begin{tabular}{|c|c|}
\hline Type de variables & $\begin{array}{c}\text { Définition d'après les questions du survey de la } \\
\text { Banque Mondiale }\end{array}$ \\
\hline $\begin{array}{c}\text { Innovation produit (Innov_Product) } \\
\text { Oui/non }\end{array}$ & $\begin{array}{c}\text { Au cours des trois dernières années, cet établissement } \\
\text { a-t-il introduit des produits ou services nouveaux ou } \\
\text { significativement améliorés ? }\end{array}$ \\
\hline $\begin{array}{c}\text { Innovation processus (Innov_Process) } \\
\text { Oui/non }\end{array}$ & $\begin{array}{c}\text { Au cours des trois dernières années, cet établissement } \\
\text { a-t-il introduit un ou plusieurs processus nouveau(x) ou } \\
\text { significativement amélioré(s), à savoir : } \\
\text { des méthodes de fabrication de produits ou d'offre de } \\
\text { services ; des méthodes logistiques, de livraison ou de } \\
\text { distribution pour les facteurs de production, les } \\
\text { produits ou les services ; ou des activités d'appui aux } \\
\text { processus ? }\end{array}$ \\
\hline Innovateur simple (Innov_Single) & $\begin{array}{c}\text { Innove dans les produits } \text { ou innove dans les processus } \\
\text { Variable construite }\end{array}$ \\
\hline Oni/non & Innove dans les produits et dans les processus \\
Oui/non & Variable construite \\
\hline
\end{tabular}

Variables indépendantes :

\begin{tabular}{|c|c|}
\hline Investissement R\&D (RRDIN) & $\begin{array}{c}\text { Au cours de la dernière année fiscale, cet } \\
\text { établissement a-t-il investi dans des activités } \\
\text { formelles de recherche et développement, que ce } \\
\text { soit en interne ou par le biais d'autres sociétés, à } \\
\text { l'exception des études de marché ? }\end{array}$ \\
\hline Pourcentage de diplômés & $\begin{array}{c}\text { Pourcentage d'employés permanents à temps } \\
\text { plein qui ont terminé leurs études secondaires }\end{array}$ \\
\hline Taille de l'entreprise & Nombre d'employés \\
\hline Industrie (Indus) & $\begin{array}{c}\text { Cette variable prend 3 modalités : Indus1 } \\
\text { (industrie manufacturière) ; Indus2 (commerce de } \\
\text { détail), Indus3 (autres services) }\end{array}$ \\
\hline
\end{tabular}

Tableau 1. La définition des variables de l'analyse économétrique 
Sur 351 firmes pour lesquelles nous avons des informations sur leur comportement d'innovation, nous avons calculé les populations d'innovateurs.

Par rapport aux données d'autres études nos proportions respectent un ordre identique : on a plus d'innovateurs de produits que de procédés, plus d'innovateurs simples que d'innovateurs complexes. La proportion d'innovateurs est presque identique à celle trouvé par Le Bas et Poussing (2014) pour le Luxembourg (50\%). Mais ni les pays ne sont identiques ni le type d'enquêtes. Aussi la comparaison devrait s'arrêter là.

\begin{tabular}{|c|c|}
\hline & $\begin{array}{c}\text { Proportion par } \\
\text { rapport à la } \\
\text { population totale } \\
(\mathbf{\%})\end{array}$ \\
\hline $\begin{array}{c}\text { Innovateur de } \\
\text { produit }^{6}\end{array}$ & 40,4 \\
\hline $\begin{array}{c}\text { Innovateur de } \\
\text { procédé }\end{array}$ & 14,4 \\
\hline $\begin{array}{c}\text { Innovateur } \\
\text { simple }\end{array}$ & 32,7 \\
\hline $\begin{array}{c}\text { Innovateur } \\
\text { complexe }\end{array}$ & 11,1 \\
\hline Total innovateur & 43,8 \\
\hline
\end{tabular}

Tableau 2. Les populations de firmes innovantes

\section{Modèles estimés et résultats}

La stratégie économétrique que nous avons suivie dépend fortement de nos données. On dispose via la Banque Mondiale d'informations sur les données d'entreprises camerounaises sur plus d'une dizaine années. Toutefois, il y a trop d'informations manquantes pour travailler sur un panel d'entreprises et avoir recours aux méthodes d'économétrie de panel. On choisit d'expliquer la décision d'innover pour une seule année. Le modèle probit en coupe s'offre alors comme le plus pertinent. L'équation estimée est :

$$
\mathrm{y}_{i}(\mathrm{t})=\sum a_{j} \cdot \mathrm{x}_{i j}(\mathrm{t})+\mathrm{u}_{i}(\mathrm{t})
$$

$\mathrm{y}_{\mathrm{i}}(\mathrm{t})$ est la probabilité que la firme i décide d'innover. Elle est fonction des régresseurs $\mathrm{x}_{\mathrm{ij}}$ directement observables sur la même période. Les coefficients $a_{j}$ doivent être estimés. Les $u_{i}(t)$ constituent les termes aléatoires dont la distribution est gaussienne. On estime cette équation pour les 4 catégories d'innovateurs. Le tableau 3 fournit les résultats.

Les résultats qui émergent sont les suivants. L'existence d'investissement de R-D n'a pas d'impact sur le comportement d'innovation à l'exception du comportement des innovateurs complexes. On trouve un impact réel des activités de connaissances à travers la proportion de diplômés. Cependant, cet impact disparaît pour les innovateurs complexes pour lesquels l'investissement de R-D devient au contraire significatif ${ }^{7}$. La taille n'a pas d'impact en général sur la propension à innover. Ce que trouve aussi Adeyeye et al. (2015) sur les entreprises nigérianes. Une exception pour le comportement d'innovation de produit. Elle a un effet significativement négatif. On peut trouver ici un effet start-ups ou du dynamisme des petites structures.

\footnotetext{
${ }^{6}$ Les innovateurs de produit et les innovateurs de procédé ne sont pas de purs innovateurs de produits et de procédés.

${ }^{7}$ Des estimations non reproduites ici avec d'autres variables ne donnent aucune information nouvelle. Ainsi, la concurrence ne marche pas, l'appartenance à un groupe n'a pas d'impact de même que l'âge de la firme. Cela ne veut évidemment pas dire que ces phénomènes ne jouent aucun rôle ; dans le cas des données et de nos spécifications ils ne sont pas opérants. 
En conséquence, l'idée conclusive générale qui vient à l'esprit est que nos deux hypothèses ne peuvent être infirmées par nos estimations.

\begin{tabular}{|c|c|c|c|c|}
\hline & $\begin{array}{c}\text { Probabilité } \\
\text { d'être } \\
\text { innovateur } \\
\text { produit }\end{array}$ & $\begin{array}{c}\text { Probabilité } \\
\text { d'être } \\
\text { innovateur de } \\
\text { procédé }\end{array}$ & $\begin{array}{c}\text { Probabilité } \\
\text { d'être } \\
\text { innovateur } \\
\text { simple }\end{array}$ & $\begin{array}{c}\text { Probabilité } \\
\text { d'être } \\
\text { Innovateur } \\
\text { complexe }\end{array}$ \\
\hline Âge & 0,0090 & 0,0084 & $-0,0072$ & 0,0083 \\
$(0,9543)$ & $(0,1783)$ & $(0,2004)$ & $(0,2188)$ \\
\hline Investissement en & 0,3202 & 0,2985 & $-0,0558$ & $\mathbf{0 , 4 9 8 7 *}$ \\
R-D & $(0,1728)$ & $(0,2703)$ & $(0,8226)$ & $(0,0688)$ \\
\hline \% de diplômés & $\mathbf{0 , 4 5 4 3 * *}$ & $\mathbf{0 , 5 2 3 3 *}$ & $\mathbf{0 , 4 7 8 6 * *}$ & 0,3511 \\
& $(0,0200)$ & $(0,0824)$ & $(0,0270)$ & $(0,2493)$ \\
\hline Taille & $\mathbf{- 0 , 0 0 2 5 * *}$ & $-0,0005$ & $-0,0007$ & $-0,0008$ \\
& $(0,0489)$ & $(0,4843)$ & $(0,4042)$ & $(0,7005)$ \\
\hline Indus 1 & 0,2681 & $-0,1228$ & 0,1063 & 0,1279 \\
& $(0,1272)$ & $(0,5890)$ & $(0,5560)$ & $(0,6039)$ \\
\hline Indus 3 & 0,0581 & $-0,2042$ & 0,0810 & $-0,0888$ \\
& $(0,7302)$ & $(0,3536)$ & $(0,6470)$ & $(0,7005)$ \\
\hline Constante & $-0,4520$ & $-16,1716$ & 14,8565 & $-16,2900$ \\
& $(0,3373)$ & $(0,1959)$ & $(0,1874)$ & $(0,2289)$ \\
\hline
\end{tabular}

Tableau 3. Les déterminants du comportement d'innovation (modèle Probit)

La variable Indus2 est prise comme référence. Pour ces estimations le nombre d'observations exploitables est $335^{\circ}$. Le pseudo R2 est très faible de l'ordre de $2 \%$. La $\mathrm{t}$ statistique est entre parenthèses. * significatif au seuil de $10 \%$, ** significatif au seuil de $5 \%$.

En résumé, le schéma standard de l'innovation produite par de la R-D est ici peu représentatif. Une explication forte est que les économies africaines à revenus intermédiaires ne disposent pas de grandes firmes ayant de départements de recherche (sur cet aspect voir le travail très documenté de Fu et al., 2018). Nos résultats sont en cohérence avec la littérature qui montre que la R-D ne joue pas toujours le rôle d'input dans les industries low tech africaines à la différence de ce qui se fait dans les économies développées. Par exemple, le travail de Goedhuys et al. (2013), sur plusieurs économies du sud dont deux économies africaines (Afrique du Sud, Tanzanie), indique que les sources de la croissance et de la productivité diffèrent fortement d'une industrie à une autre. Dans les industries alimentaires, la productivité est affectée par la qualité du management. Dans l'industrie du textile, l'importation de nouvelles machines est un facteur de croissance de la productivité à côté de la R-D. Pour ce qui est de l'industrie de l'habillement, la R-D et les activités de conception ont un impact mais en complément de la qualité du management et le licensing.

Par ailleurs, il y a tout un ensemble de formes d'innovation (associées à de nouveaux Business Models) qui sont produites sans beaucoup d'activités technologiques. Dans ce type d'espace, les barrières à l'entrée sont faibles et beaucoup d'acteurs peuvent participer (Paunov, 2013). Ce type d'innovation est approprié car bien souvent les innovateurs dans les pays africains ne peuvent avoir accès au marché des capitaux. On est donc assez vite ramené à la dynamique du secteur informel. La montée du niveau d'éducation (on le voit dans les résultats) peut favoriser ce type d'innovation (dans les TIC par exemple). Ce point est important. Il signale une possible complémentarité avec les activités de R-D. Plus généralement un examen des effets de variables d'interaction serait souhaitable $^{9}$

\footnotetext{
${ }^{8}$ On a calculé précédemment les proportions de différents innovateurs sur 351 observations.

${ }^{9}$ Les résultats d'Egbetokun et al. (2009) sur les entreprises nigérianes montrent que les interactions entre variables importent plus que les variables elles-mêmes sur l'effort d'innovation des firmes. 
On peut enfin noter que l'absence d'impact de la R-D pourrait renvoyer à la faiblesse du système de brevet (y compris les aspects juridictionnels) dans les pays africains en général et les économies à revenu intermédiaire en cohérence avec une volumineuse littérature (elle est passée en revue par Zanello et al., 2016: 14).

\section{Interprétation des résultats: des schémas pour l'innovation sans recherche- développement (R-D)}

L'approche néo-schumpetérienne de l'innovation ne fonctionne donc pas (ou pas pleinement) en économies africaines à revenu intermédiaire. D'autres modèles d'innovation sont plus opératoires, ils autorisent une lecture des résultats de notre analyse économétrique. On en retient ici trois.

\subsection{Un modèle d'innovateur atypique : market push versus market pull}

Les innovateurs qui réussissent en Afrique ont une démarche atypique par rapport au modèle d'innovateurs que retient la théorie économique de l'innovation. Les innovateurs qui réussissent s'adressent aux segments les plus pauvres de la population, créant des entreprises de type low-cost, tentent d'éviter les actions de corruption, mal endémique des économies pauvres ou à revenu intermédiaire (Christensen et al., 2017). Notons que la solution innovante n'est pas toujours technologique, elle peut relever d'un nouveau conditionnement, d'un produit de consommation associé à une forme innovante de commercialisation (voir l'exemple des pâtes au Nigéria fournit par Christensen et al. (2017)).

Pour résumer brièvement ce modèle d'innovation: ils génèrent eux-mêmes leur marché en s'adressant aux catégories les plus pauvres de la population. Pendant longtemps, on a cru que la clientèle cible pour les innovateurs était la "middle class". Modèle qui a fonctionné à l'occasion du décollage des économies asiatiques. La différence est que cette classe moyenne est peu nombreuse et pas réellement croissante en Afrique. Christensen et al. (2017) distinguent deux types de stratégies d'innovateurs en relation avec le marché. La stratégie Push repose sur des solutions qui sont imposées aux marchés. Par contre, la stratégie Pull vise à répondre aux besoins des consommateurs les plus pauvres. Les innovateurs créent leurs marchés qui servent de support à une croissance soutenable. En d'autres termes, "the most essential trait shared by the market-creating innovators we have studied is their ability to target non consumption - to sense the unmet needs that potential consumers struggle to satisfy and to develop solutions and business models that can meet them. These innovators adopt a different perspective on the world - they look for what isn't being consumed" (Christensen et al., 2017) ${ }^{10}$. Une caractéristique de cette stratégie est l'identification d'une ressource naturelle ou humaine qui pourrait être incorporée dans une nouvelle combinaison à bas coût.

\subsection{L'innovation « below the radar »}

Pendant longtemps, l'innovation a été conçue comme essentiellement technologique et produit pas les activités de R-D. On pouvait la repérer et la mesurer par les indicateurs classiques d'activités d'innovation comme les dépenses de R-D ou les quantités des brevets. On laisse alors dans l'ombre (en dehors du radar) des innovations pas technologiques, peu utilisatrices de dépenses de R-D et de faible ampleur en termes d'impacts sur les marchés. Avec Kaplinsky (2011), on désigne sous le terme d'innovation below the radar des innovations incrémentales très souvent de process faites par les entreprises dans leur activité de fabrication qui n'impliquent pas nécessairement d'investissement de R-D ni de travaux de scientifiques ou d'ingénieurs.

\footnotetext{
${ }^{10} \mathrm{Il}$ ne faudrait pas oublier une des barrières à l'innovation, la pénurie répandue de personnel qualifié est une donnée limitant la croissance dans les pays africains à revenu intermédiaire. Christensen et al. (2017) fournissent une méthodologie pour construire des projets d'innovation répondant à cette logique. 


\subsection{L'innovation frugale et innovation venant de la base}

Les innovations dans le secteur informel sont principalement frugales par nature et viennent de la base (Singh, 2017). Plusieurs catégories de ce type d'innovation ont été identifiées récemment dans la littérature en relation avec des économies pauvres en ressources. S'agissant de l'innovation Frugale, Basu et al. (2013) notent que ce type d'innovation vise à fournir de nouveaux biens adaptables, abordables et accessibles pour les consommateurs les plus pauvres et en particulier ceux des marchés émergents. Cette innovation illustre parfaitement ce que Radjou et al. (2013) désignent dans la formule : «do more with less », soit la recherche du juste nécessaire (Midler et al., 2017). Elle correspond, en fait, à un nouveau paradigme technologique au sens de Dosi (Dosi, 1982 ; Haudeville et Le Bas, 2016a, 2016b) offrant la possibilité de trouver une solution avec moins de ressources à toute une classe de problèmes en fournissant des produits robustes, solides, peu complexes en termes de technologies - ou peu sophistiqués - à des catégories sociales les plus pauvres.

On trouve dans la littérature un concept voisin de l'innovation frugale: l'innovation inclusive qui vise à répondre aux besoins des populations les plus pauvres comme une conséquence essentielle de l'innovation. S'agissant de l'Innovation venant de la base ${ }^{11}$, ce type d'innovation est encore appelée bottom up innovations. Pour Seyfang et Smith (2007), il s'agit d'innovation produite par les organisations de la société civile (communautés; ONG, coopératives). Relevant souvent d'une problématique de développement soutenable, on les trouve dans le secteur de l'énergie, l'alimentation ou le transport ${ }^{12}$. Selon Brem et Wolfram (2014), la préoccupation de responsabilité sociale des organisations et l'inventivité des populations pauvres constituent les racines de ce type d'innovation. Elles sont inclusives par destination.

La littérature sur l'innovation frugale souligne que ce type d'innovation est concentré dans les secteurs de l'alimentation, de la santé, des conditions de vie qui améliorent le bien-être des groupes de consommateurs pauvres dont les demandes sont très sensibles aux prix. Le point important est qu'elle n'a pas (encore) été beaucoup investiguée dans le contexte des économies africaines (Haudeville et Le Bas, 2016a). Ce nouveau schéma d'innovation fondé sur l'inclusion dans les normes de consommation des secteurs les plus pauvres pourrait prendre appui sur l'ingéniosité et la résilience des populations vivant sur la ligne de pauvreté. Ce nouveau réservoir d'innovation joue comme un incubateur (l'innovation serait alors plus jugaad que frugal). Toutefois, il ne faudrait pas passer trop vite sur ses difficultés de mise en œuvre. Ce modèle impose qu'on repense la conception, le re-ingéniering, la distribution des nouveaux produits (Zanello et al., 2016). Autant des barrières à la diffusion de l'innovation frugale et inclusive.

\section{Conclusion : quelques enseignements pour le processus de développement}

De façon générale, les résultats qui se dégagent de cet article ne réfutent pas les hypothèses que nous avons construites. Ainsi, le niveau de formation de la ressource humaine permet de se prononcer sur le rôle primordial de l'innovation au Cameroun. En revanche, les dépenses de R-D n'expliquent qu'une forme d'innovation ${ }^{13}$. On est donc très loin du modèle schumpetérien d'innovation dont la tradition Mansfield-Pavitt-Scherer a fait un schéma incontournable.

Ce travail n'échappe pas à certaines limites. La base statistique utilisée présente des données manquantes et la taille de l'échantillon n'est pas suffisamment représentative. Nos résultats ne peuvent sans doute pas être généralisés à toutes les économies africaines. Ces limites n’enlèvent en

\footnotetext{
${ }^{11}$ Le terme anglais consacré dans la littérature est Grassroots innovations.

${ }^{12}$ On trouve dans la littérature l'idée que des grassroots innovations » sont des innovations faites par les pauvres.

${ }^{13} \mathrm{Il}$ ne faudrait pas non plus généraliser ce résultat car par exemple Adeyeye et al. (2005) montrent à l'aide d'une enquête menée au Nigéria que la R-D interne influence les différents types d'innovation. 
rien l'intérêt de cette recherche mais incitent à construire d'autres réflexions sur les drivers de l'innovation dans les économies à revenu intermédiaire.

En termes de prolongements, la dimension géographique mériterait de plus amples investigations. On a indiqué la forte concentration des entreprises de l'échantillon autour de quelques grands pôles urbains. Une meilleure évaluation du rôle des réseaux industriels et des réseaux de connaissances devrait être faite ${ }^{14}$. Mais ce travail n'est pas facile à mener compte tenu du manque de littérature sur cette thématique dans les économies africaines. Les recherches sur l'industrialisation des pays asiatiques ont montré que les entreprises dans les économies moins développées font face à des difficultés lorsqu'elles veulent entrer dans des partenariats (avec des fournisseurs par exemple). Ce qui imposent de détenir des services ou des inputs comme des standards, des certifications, des dispositifs de propriété intellectuelle que seulement les pouvoirs publics peuvent fournir (voir par exemple Saxenian et Sabel ; 2011). Aussi, on perçoit une limite qui est que l'innovation impose des collaborations entre acteurs et que bien souvent en économies africaines les dispositifs institutionnels qui faciliteraient des interactions et/ou des transactions de ce type sont manquants ${ }^{15}$.

D'une certaine façon, c'est toute la problématique des systèmes nationaux ou locaux d'innovation qui affleure $\mathrm{ici}^{16}$. Il y a une autre question adjacente, celle de l'activité entrepreneuriale qui est à la base de l'innovation. Les barrières à cette activité dressées par les firmes en place ou les différents groupes d'intérêt constituent d'importants empêchements à l'innovation ou au développement économique (Carlsson, 2011). Il est clair que dans les économies africaines le manque d'institution tirant vers le haut la dynamique entrepreneuriale joue négativement sur la création de nouvelles idées et leur commercialisation.

En termes d'implications pour le Développement, on souligne à nouveau que l'investissement en R-D présente, quand il existe, des risques énormes dans les économies africaines. En conséquence, il reste l'exclusivité des pays riches créant davantage le fossé technologique entre le Nord et le Sud. De ce fait, les économies à revenu intermédiaire, notamment le Cameroun, n'associent pas les investissements d'innovation à la R-D.

Sans vouloir traiter au fond la question de la relation au développement, notre recherche met en évidence deux faits stylisés permettant de faire avancer la recherche. Le premier renvoie à l'idée que les innovateurs qui réussissent en Afrique ont une démarche atypique par rapport au modèle d'innovateurs que retient la théorie économique de l'innovation, ils sont market push et non market pull. Ils s'adressent aux segments les plus pauvres de la population, créant des entreprises de type low-cost, tentent d'éviter les actions de corruption, mal endémique des économies pauvres ou à revenu intermédiaire (Christensen et al., 2017). Le continent africain est le lieu d'une nouvelle génération d'entrepreneurs dont l'ingéniosité s'est affirmée dans des secteurs aussi variés que la santé, la téléphonie mobile, l'agriculture. Une seconde perspective relève de la nature même de l'innovation. L'innovation qui réussit n'est pas toujours technologique, elle peut relever d'un nouveau conditionnement d'un produit de consommation associé à une forme innovante de commercialisation (voir l'exemple des pâtes au Nigéria fournit par Christensen et al. (2017)). Cela permet de comprendre que l'innovation peut se faire sans recherche-développement et donc «below the radar ». Faut-il relier ce type d'innovation au fonctionnement du secteur informel dont on sait qu'il est important pour la survie des entreprises et des économies ? En définitive cela permet d'avancer l'idée que le développement ne nécessite pas obligatoirement une dynamique

\footnotetext{
${ }^{14}$ Pour une définition et une meilleure connaissance du fonctionnement de ces réseaux voir, entre autres, le travail de synthèse de Giuliani (2011).

${ }^{15}$ Egbetokun et al. (2009) ont montré l'importance des interactions ou des collaborations entre acteurs pour réussir l'innovation dans le contexte nigérian.

${ }^{16}$ L'étude d'Egbetokun et al. (2009) souligne l'impact potentiel de ce facteur sur la capacité d'innovation des entreprises nigérianes. 
technologique très intensive en R-D comme cela a été dans les années 1950 à 1970 (voir notamment Currie-Alder, 2016 ; Jerven, 2014 : Hirschman, 1958 ; Rosenberg, 1972).

Les principaux défis en matière d'innovation en Afrique seraient, entre autres, d'encourager la croissance inclusive, de soutenir la recherche face aux enjeux sociaux majeurs et surtout d'investir dans la formation des ressources humaines. L'accumulation des connaissances devrait conduire à une augmentation des compétences technologiques. En fait, deux voies s'offrent en matière de politique publique en faveur de l'innovation. Dans une optique plus classique, on pense que l'adoption de technologies importées permettrait aux économies africaines d'être capable de (re)développer les technologies locales. Une autre option viserait à mettre l'innovation frugale mais « africanisée » 17 au cœur du dispositif (système) national d'innovation.

\section{Bibliographie}

[ADE] ADEDAYO S. O., OJO J. O., «Family Conflict and Sustainability of Family Owned Businesses in Lagos and Ogun States, Nigeria », Journal of Business and Finance Management Research, vol.2, n, p.89-96, 2016.

[ADE] ADEYEYE A.D., JEGEDE O.O., OLUWADARE A.J., AREMU F.S., « Microlevel determinants of innovation: analysis of the Nigerian manufacturing sector », Innovation and Development http://dx.doi.org/10.1080/2157930X.2015.1047110, 2015.

[ASO] ASONGU S. A., «Knowledge Economy Gaps, Policy Syndromes and Catch-up Strategies: Fresh South Korean Lessons to Africa », AGDI Working. Paper, No. WP/14/014, 2014.

[BAR] BARNEY J. B., «Firm Resources and Sustained Competitive Advantage: a comment », Journal of Management, vol. 17, $\mathrm{n}^{\circ} 1$, p. 99-120, 1991.

[BAS] BASU R., BANERJEE P., SWEENY E., « Frugal Innovation: Core Competencies to Address Global Sustainability », Journal of Management for Global Sustainability, vol. 1, n², p.63-82, 2013.

[BRE] BREM A., WOLFRAM P., « Research and development from the bottom up -introduction of terminologies for new product development in emerging markets », Journal of Innovation and Entrepreneurship, vol. 3, n ${ }^{\circ}$, p.2-22, 2014.

[CAR] CARLSSON B., New knowledge: the driving force of innovation, entrepreneurship, and economic development. Handbook of research on innovation and entrepreneurship, 2011.

[CAS] CASADELLA V., TEMPLE L., Politiques d'innovation pour le développement des économies du Sud: regards croisés Sénégal/Cameroun, http://agritrop.cirad.fr/582715/, 2016.

[CHA] CHALEARD J-L, SANJUAN T., Géographie du développement. Territoires et mondialisation dans les Suds, Armand Colin Collection U, 2017.

[CHA] CHATAWAY J., HANLIN R., KAPLINSKY R., " Inclusive innovation: an architecture for policy development. Innovation and Development, vol.4, $\mathrm{n}^{\circ} 1$, p.33-54, 2014.

[CHR] CHRISTENSEN C. N., OJOMO E., VAN BEVER D., « Africa's new generation of innovators », Harvard Business Review, vol. 95, n¹, p.128-136, 2017.

[CUR] CURRIE-ALDER B., "The state of development studies: origins, evolution and prospects », Canadian Journal of Development Studies/Revue canadienne d'études du développement, vol.37, nº1, 5-26, 2016.

[DIA] DIAO X., MCMILLAN M., RODRIK D, The recent growth boom in developing economies: A structural change perspective, (No. w23132), National Bureau of Economic Research, 2017.

[DOS] DOSI G., « Technological paradigms and technological trajectories: a suggested interpretation of the determinants and directions of technical change », Research policy, vol.11, n³, p.147-162, 1982.

[EGB] EGBETOKUN A., SIYANBOLA W., SANNI M., OLAMADE O., ADENIYI A., IREFIN I., « What drives innovation ?: Inferences from an industry-wide survey in Nigeria », International Journal of Technology Management, vol.45, $\mathrm{n}^{\circ} 1 / 2$, p.123-140, 2009.

\footnotetext{
${ }^{17}$ C'est-à-dire débarrassée de ses références indiennes.
} 
[FAG] FAGERBERG J., "Innovation: A guide to the literature », in [FAG] FAGERBERG J., MOWERY D.C., NELSON R.R. (Eds), The Oxford Handbook of Innovation, Oxford University Press, 2005.

[FU] FU X., MOHNEN P., ZANELLO G., «Innovation and productivity in formal and informal firms in Ghana », Technological Forecasting and Social Change, vol.131, p.315-325, 2018.

[GIU] GIULIANI E., Networks of innovation, Handbook of regional innovation and growth, 155. Edward Elgar, Chelthenham, 2011.

[HAU 16a] HAUDEVILLE B., LE BAS C., «L'innovation frugale : une nouvelle opportunité pour les économies en développement? », Mondes en développement, $\mathrm{n}^{\circ} 173, \mathrm{p} .11-28,2016$.

[HAU 16b] HAUDEVILLE B., LE BAS C., «L'innovation frugale, paradigme technologique naissant ou nouveau modèle d'innovation ? », Innovations, vol.3, n 51, p. 9-25, 2016.

[HAU] HAUDEVILLE B., LE BAS C., «Développer l'innovation en Afrique et dans les PMA: construire le SNI, encadrer l'innovation frugale, multiplier les systèmes locaux », Mondes en développement, $\mathrm{n}^{\circ}$ 4, p.101-118, 2018.

[HIR] HIRSCHMAN A. O., The strategy of economic development (No. 04; HD82, H5.), 1958.

[HOB] HOBDAY M., " Firm-level innovation models: perspectives on research in developed and developing countries », Technology Analysis \& Strategic Management, vol. 17, n², p.121-14, 2005.

[JAC] JACQUEMOT P., Économie politique de l'Afrique contemporaine, Collection U, Armand Colin, 2013.

[JER] JERVEN M., « Measuring African development: past and present. Introduction to the Special Issue », Canadian Journal of Development Studies/Revue canadienne d'études du développement, vol.35, nº 1, p.1-8, 2014.

[KAP] KAPLINSKY R., "Schumacher meets Schumpeter: appropriate technology below the radar », Research Policy, vol. 40, p.193-203, 2011.

[LAZ] LAZONICK W., «The innovative firm », in Fagerberg J., Mowery D.C., Nelson R.R. (Eds), The Oxford Handbook of Innovation, Oxford University Press, 2005.

[LE] LE BAS C., POUSSING N., « Are complex innovators more persistent than single innovators? An empirical analysis of innovation persistence drivers », International Journal of Innovation Management, vol.18, $\mathrm{n}^{\circ}$ 1, 2014.

[MAN] MANSFIELD E., «Innovation, Technology and the Economy », the Selected essays of Edwin Mansfield, vol.1, 1995.

[MID] MIDLER C, JULLIEN B., LUNG Y. Innover à l'envers - Repenser la stratégie et la conception dans un monde frugal, Dunod, Paris, 2017.

[NEL] NELSON R. R., WINTER S. G. (1982), An Evolutionary Theory of Economic Change, Cambridge: Belknap Press of Harvard University Press, p. 59-65, 1982.

[PAU] PAUNOV C., «Innovation and inclusive development: a discussion of the main policy issues », OECD Science, Technology and Industry Working Papers, $\mathrm{n}^{\circ} 1,2013$.

[PAV] PAVITT K., « Sectoral patterns of technological change: toward a taxonomy and a theory», Research Policy, vol.13, no.6, p.343-373, 1984.

[PIA] PIANTA M., «Innovation and employment », In The Oxford Handbook of Innovation, [FAG] FAGERBERG J., DC Mowery and RR Nelson (Eds.), Oxford, England: Oxford University, p.568-598; 2005.

[RAD] RADJOU N., PRABHU J., AHUJA S., L'Innovation jugaad: Redevenons ingénieux!, Éditions Diateino, Paris, 2013.

[RAN] RANIS G., FEI J. C., « A Theory of Economic Development », American Economic Review, vol.51, n 4 , p.533-558, 1961.

[ROS] ROSENBERG N., Technology and American economic growth (p. 1). New York: Harper \& Row, 1972.

[SAX] SAXENIAN A., SABEL C. F., «The new Argonauts and the Rise of Venture Capital in the Periphery », Handbook of Research on Innovation and Entrepreneurship, 104, 2011.

[SCH] SCHERER F.M., Innovation and Growth. Schumpeterian Perspectives, MIT Press, Cambridge, 1991.

[SEY] SEYFANG G, SMITH A., « Grassroots innovations for sustainable development: towards a new research and policy agenda », Environmental Politics, vol.16, n², p.584-603, 2007. 
[SIN] SINGH B. (2017), « Concept of Frugality and Informal Sector Innovations in the Context of Local Development », Proceedings of International Conference on Strategies in Volatile and Uncertain Environment for Emerging Markets July 14-15, Indian Institute of Technology Delhi, New Delhi pp.573-579, 2017.

[SUM] SUMBERG J., « Systems of innovation theory and the changing architecture of agricultural research in Africa », Food Policy, vol.30, n 1, p. 21-41, 2005.

[SWA] SWANN G.M.P., The Economics of Innovation: An Introduction, Edward Elgar, Chelthenham, 2009.

[TAV] TAVASSOLI S., KARLSSON C., « Persistence of various types of innovation analyzed and explained », Research Policy, vol. 44, n¹0, p.1887-1901, 2015.

[TEE] TEECE D.J., PISANO G. (1994) « The dynamic capabilities of firms: an introduction », Working Paper $n^{\circ} W P-$ 94-103, 1994.

[TEE] TEECE D.J., PISANO G., SHUEN A., «Dynamic Capabilities and Strategic Management », Strategic Management Journal, vol.18, $\mathrm{n}^{\circ}$ 7, p.509-533, 1997.

[WER] WERNERFELT B., «A resource-based view of the firm », Strategic Management Journal, vol.5, n², p.171$180,1984$.

[WRI] WRIGHT P.M., MCCORMICK B., SHERMAN W. S., MCMAHAN G.C., « The role of human resources practices in petro-chemical refinery performance », The International Journal of Human Resource Management, vol.10, p.551-571, 1999.

[WRI] WRIGHT P.M., DUNFORD B.B., SNELL S.A., « Human resources and the resource based view of the firm », Journal of Management, vol.27, pp.701-721, 2001.

[ZAN] ZANELLO G., FU X., MOHNEN P., VENTRESCA M., « The creation and diffusion of innovation in developing countries: a systematic literature review », Journal of Economic Surveys, vol.30, n5, p.884-912, 2016.

\begin{tabular}{|l|c|}
\hline \multicolumn{1}{|c|}{ VarName } & $\begin{array}{c}\text { Moy } \\
\text { (Std Deviation) }\end{array}$ \\
\hline inno_produit & 0.404432133 \\
& $(0.4914629764)$ \\
inno_procede & 0.1440443213 \\
& $(0.3516220165)$ \\
single & 0.3268698061 \\
& $(0.4697202562)$ \\
\hline complexe & 0.1108033241 \\
& $(0.3143240854)$ \\
indus1 & 0.3324099723 \\
& $(0.4717308653)$ \\
\hline indus2 & 0.3185595568 \\
& $(0.4665644262)$ \\
indus3 & 0.3490304709 \\
& $(0.4773251881)$ \\
\hline Taille & 37.513888889 \\
firme_diplome & $(98.285269677)$ \\
& 0.1551246537 \\
R-D & $(0.3625259354)$ \\
& 1.8948863636 \\
& $(0.3071363713)$ \\
\hline
\end{tabular}

Annexe 1. Statistique descriptives des variables 